\title{
Some perturbed trapezoid inequalities for convex, $s$-convex and $t g s$-convex functions and applications
}

\author{
Mevlüt Tunç^ and Ümmügülsüm Şanal` \\ * Mustafa Kemal University, Faculty of Science and Arts, Department of Mathematics, 31000, Hatay, Turkey. \\ ^ Mustafa Kemal University, Graduate School of Natural and Applied Sciences, Department of Mathematics, 31000 , \\ Hatay, Turkey. \\ * Corresponding Author. \\ E-mail: mevluttttunc@gmail.com, mtunc@mku.edu.tr, gsanal_20@hotmail.com
}

\begin{abstract}
In this paper, the Authors establish a new identity for twice differentiable functions. Afterwards some new inequalities are presented related to perturbed trapezoid inequality for the classes of functions whose second derivatives of absolute values are convex, $s$-convex and $t g s$-convex. Last of all, applications to special means have also been presented.
\end{abstract}

2010 Mathematics Subject Classification. 26A51. 26D10, 26D15, 26E60..

Keywords. Hermite-Hadamard inequalities; convex function; s-convex function; tgs-convex function; perturbed trapezoid inequality; means..

\section{Introduction}

Definition 1. [?] A function $f: I \rightarrow \mathbb{R}$ is said to be convex on $I$ if inequality

$$
f(t u+(1-t) v) \leq t f(u)+(1-t) f(v)
$$

holds for all $u, v \in I$ and $t \in[0,1]$. We say that $f$ is concave if $(-f)$ is convex.

Geometrically, this means that if $P, Q$ and $R$ are three distinct points on the graph of $f$ with $Q$ between $P$ and $R$, then $Q$ is on or below the chord $P R$.

Definition 2. [?] Let $s \in(0,1]$. A function $f:(0, \infty] \rightarrow[0, \infty]$ is said to be $s$-convex in the second sense if

$$
f(t u+(1-t) v) \leq t^{s} f(u)+(1-t)^{s} f(v),
$$

for all $u, v \in(0, b]$ and $t \in[0,1]$. This class of $s$-convex functions is usually denoted by $K_{s}^{2}$.

Certainly, $s$-convexity means just ordinary convexity when $s=1$.

Definition 3. [?] A function $f: I \rightarrow \mathbb{R}$ is said to be $\operatorname{tgs}$-convex on $I$ if inequality

$$
f(t u+(1-t) v) \leq t(1-t)[f(u)+f(v)]
$$

holds for all $u, v \in I$ and $t \in(0,1)$. We say that $f$ is tgs-concave if $(-f)$ is tgs-convex. 
Theorem 1. The Hermite-Hadamard inequality: Let $f: I \subseteq \mathbb{R} \rightarrow \mathbb{R}$ be a convex function and $u, v \in I$ with $u<v$. The following double inequality:

$$
f\left(\frac{u+v}{2}\right) \leq \frac{1}{v-u} \int_{u}^{v} f(x) d x \leq \frac{f(u)+f(v)}{2}
$$

is known in the literature as Hadamard's inequality (or Hermite-Hadamard inequality) for convex functions. If $f$ is a positive concave function, then the inequality is reversed.

Theorem 2. [?] Suppose that $f:[0, \infty) \rightarrow[0, \infty)$ is an $s$-convex function in the second sense, where $s \in(0,1]$, and let $a, b \in[0, \infty), a<b$. If $f \in L_{1}([0,1])$, then the following inequalities hold:

$$
2^{s-1} f\left(\frac{u+v}{2}\right) \leq \frac{1}{v-u} \int_{u}^{v} f(x) d x \leq \frac{f(u)+f(v)}{s+1} .
$$

The constant $k=\frac{1}{s+1}$ is the best possible in the second inequality in (??). The above inequalities are sharp. If $f$ is an $s$-concave function in the second sense, then the inequality is reversed.

For recent results and generalizations concerning Hadamard's inequality and concepts of convexity and $s$-convexity see [?]-[?], [?]-[?] and the references therein.

In the literature [?]-[?] on numerical integration, the following estimation is well known as the trapezoid inequality:

$$
\left|\int_{u}^{v} f(x) d x-\frac{1}{2}(v-u)(f(u)+f(v))\right| \leq \frac{1}{12} M_{2}(v-u)^{3},
$$

where $f:[u, v] \rightarrow \mathbb{R}$ is supposed to be twice differentiable on the interval $(u, v)$, with the second derivative bounded on $(u, v)$ by $M_{2}=\sup _{x \in(u, v)}\left|f^{\prime \prime}(x)\right|<+\infty$.

For the perturbed trapezoid inequality, Dragomir et al. [?] obtained the following inequality by an application of the Grüss inequality:

$$
\begin{aligned}
& \left|\int_{u}^{v} f(x) d x-\frac{1}{2}(v-u)(f(u)+f(v))+\frac{1}{12}(v-u)^{2}\left(f^{\prime}(v)-f^{\prime}(u)\right)\right| \\
\leq & \frac{1}{32}\left(\Gamma_{2}-\gamma_{2}\right)(v-u)^{3},
\end{aligned}
$$

where $f$ is supposed to be twice differentiable on the interval $(u, v)$, with the second derivative bounded on $(u, v)$ by $\Gamma_{2}=\sup _{x \in(u, v)} f^{\prime \prime}(x)<+\infty$ and $\gamma_{2}=\inf _{x \in(u, v)} f^{\prime \prime}(x)>-\infty$.

Throughout this paper we will use the following notations and conventions. Let $J=[0, \infty) \subset$ $\mathbb{R}=(-\infty,+\infty)$, and $u, v \in J$ with $0<u<v$ and $f^{\prime} \in L[u, v]$ and

$$
\begin{aligned}
A(u, v) & =\frac{u+v}{2}, G(u, v)=\sqrt{u v}, H(u, v)=\frac{2 u v}{u+v} \\
L(u, v) & =\frac{v-u}{\ln v-\ln u}, u \neq v
\end{aligned}
$$

be the arithmetic mean, geometric mean, harmonic mean, logarithmic mean for $u, v>0$ respectively.

The aim of this paper is to establish some results connected with the perturbed trapezoid inequality as well as to apply them for some elementary inequalities for real numbers and in numerical integration. 


\section{Main Results}

We begin with the following lemma.

Lemma 1. Let $f: I^{\circ} \subseteq R \rightarrow R$ be a differentiable mapping on $I^{\circ}, a, b \in I^{\circ}$ with $a<b$. If $f^{\prime \prime} \in L[a, b]$, then the following equality holds:

$$
\begin{aligned}
& \int_{a}^{b} f(x) d x-\frac{1}{2}(b-a)(f(a)+f(b))+\frac{5}{4}(b-a)^{2}\left(f^{\prime}(b)-f^{\prime}(a)\right) \\
= & \frac{(b-a)^{3}}{4} \int_{0}^{1}(t+1)^{2}\left[f^{\prime \prime}(t a+(1-t) b)+f^{\prime \prime}(t b+(1-t) a)\right] d t
\end{aligned}
$$

Proof. It suffices to note that

$$
\begin{aligned}
I_{1}= & \int_{0}^{1}(t+1)^{2} f^{\prime \prime}(t a+(1-t) b) d t \\
= & \left.(t+1)^{2} \frac{f^{\prime}(t a+(1-t) b)}{a-b}\right|_{0} ^{1}-\frac{2}{a-b} \int_{0}^{1}(t+1) f^{\prime}(t a+(1-t) b) d t \\
= & \frac{4 f^{\prime}(a)-f^{\prime}(b)}{a-b}-\frac{2}{a-b} \int_{0}^{1}(t+1) f^{\prime}(t a+(1-t) b) d t \\
= & \frac{4 f^{\prime}(a)-f^{\prime}(b)}{a-b}-\frac{2}{a-b}\left[\left.(t+1) \frac{f(t a+(1-t) b)}{a-b}\right|_{0} ^{1}\right. \\
& \left.-\frac{1}{a-b} \int_{0}^{1} f(t a+(1-t) b) d t\right] \\
= & \frac{4 f^{\prime}(a)-f^{\prime}(b)}{a-b}-\frac{2}{a-b}\left[\frac{2 f(a)-f(b)}{a-b}-\frac{1}{a-b} \int_{0}^{1} f(t a+(1-t) b) d t\right] \\
= & \frac{4 f^{\prime}(a)-f^{\prime}(b)}{a-b}-\frac{4 f(a)-2 f(b)}{(b-a)^{2}}+\frac{2}{(b-a)^{2}} \int_{0}^{1} f(t a+(1-t) b) d t
\end{aligned}
$$


and

$$
\begin{aligned}
I_{2}= & \int_{0}^{1}(t+1)^{2} f^{\prime \prime}(t b+(1-t) a) d t \\
= & \left.(t+1)^{2} \frac{f^{\prime}(t b+(1-t) a)}{b-a}\right|_{0} ^{1}-\frac{2}{b-a} \int_{0}^{1}(t+1) f^{\prime}(t b+(1-t) a) d t \\
= & \frac{4 f^{\prime}(b)-f^{\prime}(a)}{b-a}-\frac{2}{b-a} \int_{0}^{1}(t+1) f^{\prime}(t b+(1-t) a) d t \\
= & \frac{4 f^{\prime}(b)-f^{\prime}(a)}{b-a}-\frac{2}{b-a}\left[\left.(t+1) \frac{f(t b+(1-t) a)}{b-a}\right|_{0} ^{1}\right. \\
& \left.-\frac{1}{b-a} \int_{0}^{1} f(t b+(1-t) a) d t\right] \\
= & \frac{4 f^{\prime}(b)-f^{\prime}(a)}{b-a}-\frac{2}{b-a}\left[\frac{2 f(b)-f(a)}{b-a}-\frac{1}{b-a} \int_{0}^{1} f(t b+(1-t) a) d t\right] \\
= & \frac{4 f^{\prime}(b)-f^{\prime}(a)}{b-a}-\frac{4 f(b)-2 f(a)}{(b-a)^{2}}+\frac{2}{(b-a)^{2}} \int_{0}^{1} f(t b+(1-t) a) d t .
\end{aligned}
$$

If we collect $I_{1}$ and $I_{2}$

$$
\begin{aligned}
I_{1}+I_{2}= & \int_{0}^{1}(t+1)^{2}\left[f^{\prime \prime}(t a+(1-t) b)+f^{\prime \prime}(t b+(1-t) a)\right] d t \\
= & -\frac{4 f^{\prime}(a)-f^{\prime}(b)}{b-a}+\frac{4 f^{\prime}(b)-f^{\prime}(a)}{b-a}-\frac{4 f(a)-2 f(b)+4 f(b)-2 f(a)}{(b-a)^{2}} \\
& +\frac{2}{(b-a)^{2}} \int_{0}^{1} f(t a+(1-t) b) d t+\frac{2}{(b-a)^{2}} \int_{0}^{1} f(t b+(1-t) a) d t \\
= & \frac{5\left(f^{\prime}(b)-f^{\prime}(a)\right)}{b-a}-\frac{2(f(a)+f(b))}{(b-a)^{2}}+\frac{2}{(b-a)^{2}} \cdot \frac{1}{a-b} \int_{b}^{a} f(x) d x \\
& +\frac{2}{(b-a)^{3}} \int_{a}^{b} f(x) d x \\
= & \frac{5\left(f^{\prime}(b)-f^{\prime}(a)\right)}{b-a}-\frac{2(f(a)+f(b))}{(b-a)^{2}}+\frac{4}{(b-a)^{3}} \int_{a}^{b} f(x) d x
\end{aligned}
$$

so

$$
\begin{aligned}
& \int_{a}^{b} f(x) d x-\frac{1}{2}(b-a)(f(a)+f(b))+\frac{5}{4}(b-a)^{2}\left(f^{\prime}(b)-f^{\prime}(a)\right) \\
= & \frac{(b-a)^{3}}{4} \int_{0}^{1}(t+1)^{2}\left[f^{\prime \prime}(t a+(1-t) b)+f^{\prime \prime}(t b+(1-t) a)\right] d t
\end{aligned}
$$

The proof is done. 
Remark 1. On using the change of the variable $x=t a+(1-t) b, t \in[0,1]$, equality (??) can be written as

$$
\begin{aligned}
& \int_{a}^{b} f(x) d x-\frac{1}{2}(b-a)(f(a)+f(b))+\frac{5}{4}(b-a)^{2}\left(f^{\prime}(b)-f^{\prime}(a)\right) \\
= & \frac{(b-a)}{4} \int_{a}^{b}(x+a-2 b)^{2}\left(f^{\prime \prime}(x)+f^{\prime \prime}(a+b-x)\right) d x .
\end{aligned}
$$

Theorem 3. Let $f: I^{\circ} \subseteq R \rightarrow R$ be a differentiable mapping on $I^{\circ}, a, b \in I^{\circ}$ with $a<b$. If $\left|f^{\prime \prime}\right|$ is convex on $[a, b]$, then the following inequality holds:

$$
\begin{aligned}
& \left|\int_{a}^{b} f(x) d x-\frac{1}{2}(b-a)(f(a)+f(b))+\frac{5}{4}(b-a)^{2}\left(f^{\prime}(b)-f^{\prime}(a)\right)\right| \\
\leq & \frac{7}{12}(b-a)^{3}\left(\left|f^{\prime \prime}(a)\right|+\left|f^{\prime \prime}(b)\right|\right) .
\end{aligned}
$$

Proof. Using Lemma ??, it follows that

$$
\begin{aligned}
& \left|\int_{a}^{b} f(x) d x-\frac{1}{2}(b-a)(f(a)+f(b))+\frac{5}{4}(b-a)^{2}\left(f^{\prime}(b)-f^{\prime}(a)\right)\right| \\
= & \left|\frac{(b-a)^{3}}{4} \int_{0}^{1}(t+1)^{2}\left[f^{\prime \prime}(t a+(1-t) b)+f^{\prime \prime}(t b+(1-t) a)\right] d t\right| \\
\leq & \frac{(b-a)^{3}}{4} \int_{0}^{1}(t+1)^{2}\left(\left|f^{\prime \prime}(t a+(1-t) b)\right|+\left|f^{\prime \prime}(t b+(1-t) a)\right|\right) d t \\
\leq & \frac{(b-a)^{3}}{4} \int_{0}^{1}(t+1)^{2}\left(t\left|f^{\prime \prime}(a)\right|+(1-t)\left|f^{\prime \prime}(b)\right|\right. \\
& \left.+t\left|f^{\prime \prime}(b)\right|+(1-t)\left|f^{\prime \prime}(a)\right|\right) d t \\
\leq & \frac{(b-a)^{3}}{4}\left[\left|f^{\prime \prime}(a)\right|+\left|f^{\prime \prime}(b)\right|\right] \int_{0}^{1}(t+1)^{2} d t \\
\leq & \frac{(b-a)^{3}}{4} \frac{7}{3}\left[\left|f^{\prime \prime}(a)\right|+\left|f^{\prime \prime}(b)\right|\right] .
\end{aligned}
$$

The proof is completed.

Theorem 4. Let $s \in(0,1]$ and $f: I^{\circ} \subseteq R \rightarrow R$ be a differentiable mapping on $I^{\circ}, a, b \in I^{\circ}$ with $a<b$. If $\left|f^{\prime \prime}\right|$ is $s$-convex on $[a, b]$, then the following inequality holds:

$$
\begin{aligned}
& \left|\int_{a}^{b} f(x) d x-\frac{1}{2}(b-a)(f(a)+f(b))+\frac{5}{4}(b-a)^{2}\left(f^{\prime}(b)-f^{\prime}(a)\right)\right| \\
\leq & \frac{(b-a)^{3}}{4} \frac{5 s^{2}+23 s+28}{s^{3}+6 s^{2}+11 s+6}\left(\left|f^{\prime \prime}(a)\right|+\left|f^{\prime \prime}(b)\right|\right) .
\end{aligned}
$$


Proof. Using Lemma ?? and Definition ??, it follows that

$$
\begin{aligned}
& \left|\int_{a}^{b} f(x) d x-\frac{1}{2}(b-a)(f(a)+f(b))+\frac{5}{4}(b-a)^{2}\left(f^{\prime}(b)-f^{\prime}(a)\right)\right| \\
\leq & \frac{(b-a)^{3}}{4} \int_{0}^{1}(t+1)^{2}\left(\left|f^{\prime \prime}(t a+(1-t) b)\right|+\left|f^{\prime \prime}(t b+(1-t) a)\right|\right) d t \\
\leq & \frac{(b-a)^{3}}{4} \int_{0}^{1}(t+1)^{2}\left(t^{s}\left|f^{\prime \prime}(a)\right|+(1-t)^{s}\left|f^{\prime \prime}(b)\right|\right. \\
& \left.+t^{s}\left|f^{\prime \prime}(b)\right|+(1-t)^{s}\left|f^{\prime \prime}(a)\right|\right) d t \\
\leq & \frac{(b-a)^{3}}{4}\left(\left|f^{\prime \prime}(a)\right|+\left|f^{\prime \prime}(b)\right|\right)\left[\int_{0}^{1}(t+1)^{2} t^{s} d t+\int_{0}^{1}(t+1)^{2}(1-t)^{s} d t\right] \\
\leq & \frac{(b-a)^{3}}{4}\left(\left|f^{\prime \prime}(a)\right|+\left|f^{\prime \prime}(b)\right|\right)\left[\frac{4 s^{2}+16 s+14}{s^{3}+6 s^{2}+11 s+6}+\frac{s^{2}+7 s+14}{s^{3}+6 s^{2}+11 s+6}\right] \\
\leq & \frac{(b-a)^{3}}{4}\left(\frac{5 s^{2}+23 s+28}{s^{3}+6 s^{2}+11 s+6}\right)\left(\left|f^{\prime \prime}(a)\right|+\left|f^{\prime \prime}(b)\right|\right) .
\end{aligned}
$$

Further, since

$$
\begin{aligned}
\int_{0}^{1}(t+1)^{2} t^{s} d t & =\frac{4 s^{2}+16 s+14}{s^{3}+6 s^{2}+11 s+6} \\
\int_{0}^{1}(t+1)^{2}(1-t)^{s} d t & =\frac{s^{2}+7 s+14}{s^{3}+6 s^{2}+11 s+6}
\end{aligned}
$$

a combination of (??) and (??) immediately gives the required inequality (??).

Theorem 5. Let $f: I^{\circ} \subseteq R \rightarrow R$ be a differentiable mapping on $I^{\circ}, a, b \in I^{\circ}$ with $a<b$. If $\left|f^{\prime \prime}\right|$ is $t g s$-convex on $[a, b]$, then the following inequality holds:

$$
\begin{aligned}
& \left|\int_{a}^{b} f(x) d x-\frac{1}{2}(b-a)(f(a)+f(b))+\frac{5}{4}(b-a)^{2}\left(f^{\prime}(b)-f^{\prime}(a)\right)\right| \\
\leq & \frac{23(b-a)^{3}}{120}\left[\left|f^{\prime \prime}(a)\right|+\left|f^{\prime \prime}(b)\right|\right] .
\end{aligned}
$$


Proof. Using Lemma ?? and Definition ??, it follows that

$$
\begin{aligned}
& \left|\int_{a}^{b} f(x) d x-\frac{1}{2}(b-a)(f(a)+f(b))+\frac{5}{4}(b-a)^{2}\left(f^{\prime}(b)-f^{\prime}(a)\right)\right| \\
\leq & \frac{(b-a)^{3}}{4} \int_{0}^{1}(t+1)^{2}\left(\left|f^{\prime \prime}(t a+(1-t) b)\right|+\left|f^{\prime \prime}(t b+(1-t) a)\right|\right) d t \\
\leq & \frac{(b-a)^{3}}{4} \int_{0}^{1}(t+1)^{2}\left(\left(t(1-t)\left|f^{\prime \prime}(a)\right|+t(1-t)\left|f^{\prime \prime}(b)\right|\right)\right. \\
& \left.+\left(t(1-t)\left|f^{\prime \prime}(b)\right|+t(1-t)\left|f^{\prime \prime}(a)\right|\right) d t\right) \\
\leq & \frac{(b-a)^{3}}{4} \int_{0}^{1} 2(t+1)^{2} t(1-t)\left[\left|f^{\prime \prime}(a)\right|+\left|f^{\prime \prime}(b)\right|\right] d t \\
\leq & \frac{(b-a)^{3}\left[\left|f^{\prime \prime}(a)\right|+\left|f^{\prime \prime}(b)\right|\right]}{2} \int_{0}^{1}(t+1)^{2} t(1-t) d t \\
\leq & \frac{23(b-a)^{3}}{120}\left[\left|f^{\prime \prime}(a)\right|+\left|f^{\prime \prime}(b)\right|\right] .
\end{aligned}
$$

The proof is completed.

Theorem 6. Let $f: I^{\circ} \subseteq R \rightarrow R$ be a differentiable mapping on $I^{\circ}, a, b \in I^{\circ}$ with $a<b$, and let $p>1$ with $1 / p+1 / q=\overline{1}$. If the mapping $\left|f^{\prime \prime}\right|^{q}$ is convex on $[a, b]$, then the following inequality holds:

$$
\begin{aligned}
& \left|\int_{a}^{b} f(x) d x-\frac{1}{2}(b-a)(f(a)+f(b))+\frac{5}{4}(b-a)^{2}\left(f^{\prime}(b)-f^{\prime}(a)\right)\right| \\
& \leq \quad \frac{(b-a)^{3}}{2}\left(\frac{2^{2 p+1}-1}{2 p+1}\right)^{\frac{1}{p}}\left(\frac{\left|f^{\prime \prime}(a)\right|^{q}+\left|f^{\prime \prime}(b)\right|^{q}}{2}\right)^{\frac{1}{q}} .
\end{aligned}
$$

Proof. Using Lemma ?? and Hölder's integral inequality, we establish

$$
\begin{aligned}
& \left|\int_{a}^{b} f(x) d x-\frac{1}{2}(b-a)(f(a)+f(b))+\frac{5}{4}(b-a)^{2}\left(f^{\prime}(b)-f^{\prime}(a)\right)\right| \\
\leq & \frac{(b-a)^{3}}{4}\left[\int_{0}^{1}|t+1|^{2}\left|f^{\prime \prime}(t a+(1-t) b)\right| d t\right. \\
& \left.+\int_{0}^{1}|t+1|^{2}\left|f^{\prime \prime}(t b+(1-t) a)\right| d t\right] \\
\leq & \frac{(b-a)^{3}}{4}\left[\left(\int_{0}^{1}|t+1|^{2 p} d t\right)^{\frac{1}{p}}\left(\int_{0}^{1}\left|f^{\prime \prime}(t a+(1-t) b)\right|^{q} d t\right)^{\frac{1}{q}}\right. \\
& \left.+\left(\int_{0}^{1}|t+1|^{2 p} d t\right)^{\frac{1}{p}}\left(\int_{0}^{1}\left|f^{\prime \prime}(t b+(1-t) a)\right|^{q} d t\right)^{\frac{1}{q}}\right] \\
\leq & \frac{(b-a)^{3}}{2}\left(\frac{2^{2 p+1}-1}{2 p+1}\right)^{\frac{1}{p}}\left(\frac{\left|f^{\prime \prime}(a)\right|^{q}+\left|f^{\prime \prime}(b)\right|^{q}}{2}\right)^{\frac{1}{q}}
\end{aligned}
$$


where $1 / p+1 / q=1$. Using the convexity of $\left|f^{\prime \prime}\right|^{q}$, we have

$$
\begin{aligned}
& \int_{0}^{1}\left|f^{\prime \prime}(t a+(1-t) b)\right|^{q} d t \\
\leq & \int_{0}^{1}\left[t\left|f^{\prime \prime}(a)\right|^{q}+(1-t)\left|f^{\prime \prime}(b)\right|^{q}\right] d t=\frac{\left|f^{\prime \prime}(a)\right|^{q}+\left|f^{\prime \prime}(b)\right|^{q}}{2} \\
& \int_{0}^{1}\left|f^{\prime \prime}(t b+(1-t) a)\right|^{q} d t \\
\leq & \int_{0}^{1}\left[t\left|f^{\prime \prime}(b)\right|^{q}+(1-t)\left|f^{\prime \prime}(a)\right|^{q}\right] d t=\frac{\left|f^{\prime \prime}(a)\right|^{q}+\left|f^{\prime \prime}(b)\right|^{q}}{2}
\end{aligned}
$$

Further, since

$$
\int_{0}^{1}|t+1|^{2 p} d t=\int_{0}^{1}(t+1)^{2 p} d t=\int_{1}^{2} u^{2 p} d u=\left.\frac{u^{2 p+1}}{2 p+1}\right|_{1} ^{2}=\frac{2^{2 p+1}-1}{2 p+1}
$$

a combination of (??)-(??) immediately gives the required inequality (??).

Theorem 7. Let $s \in(0,1]$ and $f: I^{\circ} \subseteq R \rightarrow R$ be a differentiable mapping on $I^{\circ}, a, b \in I^{\circ}$ with $a<b$, and let $p, q>1$ with $1 / p+1 / q=1$. If the mapping $\left|f^{\prime \prime}\right|^{q}$ is $s$-convex on $[a, b]$, then the following inequality holds:

$$
\begin{aligned}
& \quad\left|\int_{a}^{b} f(x) d x-\frac{1}{2}(b-a)(f(a)+f(b))+\frac{5}{4}(b-a)^{2}\left(f^{\prime}(b)-f^{\prime}(a)\right)\right| \\
& \leq \frac{(b-a)^{3}}{4}\left(\frac{2^{2 p+1}-1}{2 p+1}\right)^{\frac{1}{p}} \\
& \quad \times\left\{\left(\frac{\left|f^{\prime \prime}(a)\right|^{q}}{s+1}+\frac{\left|f^{\prime \prime}(b)\right|^{q} \Gamma(s+1)}{\Gamma(s+2)}\right)^{\frac{1}{q}}+\left(\frac{\left|f^{\prime \prime}(b)\right|^{q}}{s+1}+\frac{\left|f^{\prime \prime}(a)\right|^{q} \Gamma(s+1)}{\Gamma(s+2)}\right)^{\frac{1}{q}}\right\} .
\end{aligned}
$$


Proof. Using Lemma ??, Definition ?? and Hölder's integral inequality, we get

$$
\begin{aligned}
& \left|\int_{a}^{b} f(x) d x-\frac{1}{2}(b-a)(f(a)+f(b))+\frac{5}{4}(b-a)^{2}\left(f^{\prime}(b)-f^{\prime}(a)\right)\right| \\
& \leq \frac{(b-a)^{3}}{4}\left[\int_{0}^{1}|t+1|^{2}\left|f^{\prime \prime}(t a+(1-t) b)\right| d t\right. \\
& \left.+\int_{0}^{1}|t+1|^{2}\left|f^{\prime \prime}(t b+(1-t) a)\right| d t\right] \\
& \leq \frac{(b-a)^{3}}{4}\left[\left(\int_{0}^{1}|t+1|^{2 p} d t\right)^{\frac{1}{p}}\left(\int_{0}^{1}\left|f^{\prime \prime}(t a+(1-t) b)\right|^{q} d t\right)^{\frac{1}{q}}\right. \\
& \left.+\left(\int_{0}^{1}|t+1|^{2 p} d t\right)^{\frac{1}{p}}\left(\int_{0}^{1}\left|f^{\prime \prime}(t b+(1-t) a)\right|^{q} d t\right)^{\frac{1}{q}}\right] \\
& \leq \frac{(b-a)^{3}}{4}\left(\frac{2^{2 p+1}-1}{2 p+1}\right)^{\frac{1}{p}}\left[\left(\int_{0}^{1}\left(t^{s}\left|f^{\prime \prime}(a)\right|^{q}+(1-t)^{s}\left|f^{\prime \prime}(b)\right|^{q}\right) d t\right)^{\frac{1}{q}}\right. \\
& \left.+\left(\int_{0}^{1}\left(t^{s}\left|f^{\prime \prime}(b)\right|^{q}+(1-t)^{s}\left|f^{\prime \prime}(a)\right|^{q}\right) d t\right)^{\frac{1}{q}}\right] \\
& \leq \frac{(b-a)^{3}}{4}\left(\frac{2^{2 p+1}-1}{2 p+1}\right)^{\frac{1}{p}}\left[\left(\left|f^{\prime \prime}(a)\right|^{q} \int_{0}^{1} t^{s} d t+\left|f^{\prime \prime}(b)\right|^{q} \int_{0}^{1}(1-t)^{s} d t\right)^{\frac{1}{q}}\right. \\
& \left.+\left(\left|f^{\prime \prime}(b)\right|^{q} \int_{0}^{1} t^{s} d t+\left|f^{\prime \prime}(a)\right|^{q} \int_{0}^{1}(1-t)^{s} d t\right)^{\frac{1}{q}}\right] \\
& \leq \frac{(b-a)^{3}}{4}\left(\frac{2^{2 p+1}-1}{2 p+1}\right)^{\frac{1}{p}}\left[\left(\frac{\left|f^{\prime \prime}(a)\right|^{q}}{s+1}+\frac{\left|f^{\prime \prime}(b)\right|^{q} \Gamma(s+1)}{\Gamma(s+2)}\right)^{\frac{1}{q}}\right. \\
& \left.+\left(\frac{\left|f^{\prime \prime}(b)\right|^{q}}{s+1}+\frac{\left|f^{\prime \prime}(a)\right|^{q} \Gamma(s+1)}{\Gamma(s+2)}\right)^{\frac{1}{q}}\right]
\end{aligned}
$$

Further, since

$$
\begin{aligned}
\int_{0}^{1} t^{s} d t & =\frac{1}{s+1} \\
\int_{0}^{1}(1-t)^{s} d t & =\frac{\Gamma(s+1)}{\Gamma(s+2)}
\end{aligned}
$$

a combination of (??) and (??) immediately gives the required inequality (??).

Theorem 8. Let $f: I^{\circ} \subseteq R \rightarrow R$ be a differentiable mapping on $I^{\circ}, a, b \in I^{\circ}$ with $a<b$, and let $p>1$ with $1 / p+1 / q=1$. If the mapping $\left|f^{\prime \prime}\right|^{q}$ is $\operatorname{tgs}$-convex on $[a, b]$, then the following inequality 
holds:

$$
\begin{aligned}
& \left|\int_{a}^{b} f(x) d x-\frac{1}{2}(b-a)(f(a)+f(b))+\frac{5}{4}(b-a)^{2}\left(f^{\prime}(b)-f^{\prime}(a)\right)\right| \\
& \leq \frac{(b-a)^{3}}{2}\left(\frac{1}{6}\right)^{\frac{1}{p}}\left(\frac{2^{2 p+1}-1}{2 p+1}\right)^{\frac{1}{p}}\left[\left|f^{\prime \prime}(a)\right|^{q}+\left|f^{\prime \prime}(b)\right|^{q}\right]^{\frac{1}{q}} .
\end{aligned}
$$

Proof. Using Lemma ??, Definition ?? and Hölder's integral inequality, we get

$$
\begin{aligned}
& \left|\int_{a}^{b} f(x) d x-\frac{1}{2}(b-a)(f(a)+f(b))+\frac{5}{4}(b-a)^{2}\left(f^{\prime}(b)-f^{\prime}(a)\right)\right| \\
\leq & \frac{(b-a)^{3}}{4}\left[\int_{0}^{1}|t+1|^{2}\left|f^{\prime \prime}(t a+(1-t) b)\right| d t\right. \\
& \left.+\int_{0}^{1}|t+1|^{2}\left|f^{\prime \prime}(t b+(1-t) a)\right| d t\right] \\
\leq & \frac{(b-a)^{3}}{4}\left[\left(\int_{0}^{1}|t+1|^{2 p} d t\right)^{\frac{1}{p}}\left(\int_{0}^{1}\left|f^{\prime \prime}(t a+(1-t) b)\right|^{q} d t\right)^{\frac{1}{q}}\right. \\
& \left.+\left(\int_{0}^{1}|t+1|^{2 p} d t\right)^{\frac{1}{p}}\left(\int_{0}^{1}\left|f^{\prime \prime}(t b+(1-t) a)\right|^{q} d t\right)^{\frac{1}{q}}\right] \\
\leq & \frac{(b-a)^{3}}{4}\left(\frac{2^{2 p+1}-1}{2 p+1}\right)^{\frac{1}{p}}\left[\left(\int_{0}^{1}\left(t(1-t)\left|f^{\prime \prime}(a)\right|^{q}+t(1-t)\left|f^{\prime \prime}(b)\right|^{q}\right) d t\right)^{\frac{1}{q}}\right. \\
& \left.+\left(\int_{0}^{1}\left(t(1-t)\left|f^{\prime \prime}(b)\right|^{q}+t(1-t)\left|f^{\prime \prime}(a)\right|^{q}\right) d t\right)^{\frac{1}{q}}\right] \\
\leq & \frac{(b-a)^{3}}{2}\left(\frac{2^{2 p+1}-1}{2 p+1}\right)^{\frac{1}{p}}\left[\left|f^{\prime \prime}(a)\right|^{q}+\left|f^{\prime \prime}(b)\right|^{q}\right]^{\frac{1}{q}}\left(\int_{0}^{1} t(1-t) d t\right)^{\frac{1}{q}} \\
\leq & \frac{(b-a)^{3}}{2}\left(\frac{1}{6}\right)^{\frac{1}{p}}\left(\frac{2^{2 p+1}-1}{2 p+1}\right)^{\frac{1}{p}}\left[\left|f^{\prime \prime}(a)\right|^{q}+\left|f^{\prime \prime}(b)\right|^{q}\right]^{\frac{1}{q}} \cdot
\end{aligned}
$$

The proof is completed.

Theorem 9. Let $f: I^{\circ} \subseteq R \rightarrow R$ be a differentiable mapping on $I^{\circ}, a, b \in I^{\circ}$ with $a<b$, and let $p, q>1$ with $1 / p+1 / q=1$. If the mapping $\left|f^{\prime \prime}\right|^{p}$ is convex on $[a, b]$, then the following inequality 
holds:

$$
\begin{aligned}
& \left|\int_{a}^{b} f(x) d x-\frac{1}{2}(b-a)(f(a)+f(b))+\frac{5}{4}(b-a)^{2}\left(f^{\prime}(b)-f^{\prime}(a)\right)\right| \\
& \leq \frac{(b-a)^{3}}{4}\left(\frac{7}{3}\right)^{1-\frac{1}{p}}\left\{\left(\frac{17\left|f^{\prime \prime}(a)\right|^{p}+11\left|f^{\prime \prime}(b)\right|^{p}}{12}\right)^{\frac{1}{p}}\right. \\
& \left.\quad+\left(\frac{17\left|f^{\prime \prime}(b)\right|^{p}+11\left|f^{\prime \prime}(a)\right|^{p}}{12}\right)^{\frac{1}{p}}\right\}
\end{aligned}
$$

Proof. Using Lemma ?? and power mean integral inequality, we obtain

$$
\begin{aligned}
& \left|\int_{a}^{b} f(x) d x-\frac{1}{2}(b-a)(f(a)+f(b))+\frac{5}{4}(b-a)^{2}\left(f^{\prime}(b)-f^{\prime}(a)\right)\right| \\
\leq & \frac{(b-a)^{3}}{4} \int_{0}^{1}|t+1|^{2}\left|f^{\prime \prime}(t a+(1-t) b)+f^{\prime \prime}(t b+(1-t) a)\right| d t \\
\leq & \frac{(b-a)^{3}}{4}\left(\int_{0}^{1}|t+1|^{2} d t\right)^{1-\frac{1}{p}} \\
& \left\{\left(\int_{0}^{1}(t+1)^{2}\left(t\left|f^{\prime \prime}(a)\right|^{p}+(1-t)\left|f^{\prime \prime}(b)\right|^{p}\right) d t\right)^{\frac{1}{p}}\right. \\
& \left.+\left(\int_{0}^{1}(t+1)^{2}\left(t\left|f^{\prime \prime}(b)\right|^{p}+(1-t)\left|f^{\prime \prime}(a)\right|^{p}\right) d t\right)^{\frac{1}{p}}\right\} \\
\leq & \frac{(b-a)^{3}}{4}\left(\frac{7}{3}\right)^{1-\frac{1}{p}}\left\{\left(\frac{17\left|f^{\prime \prime}(a)\right|^{p}+11\left|f^{\prime \prime}(b)\right|^{p}}{12}\right)^{\frac{1}{p}}\right. \\
& +\left(\frac{17\left|f^{\prime \prime}(b)\right|^{p}+11\left|f^{\prime \prime}(a)\right|^{p}}{12}\right)^{\frac{1}{p}} .
\end{aligned}
$$

The proof is completed.

Theorem 10. Let $s \in(0,1]$ and $f: I^{\circ} \subseteq R \rightarrow R$ be a differentiable mapping on $I^{\circ}, a, b \in I^{\circ}$ with $a<b$, and let $p>1$ with $1 / p+1 / q=\overline{1}$. If the new mapping $\left|f^{\prime \prime}\right|^{p}$ is convex on $[a, b]$, then the 
following inequality holds:

$$
\begin{aligned}
& \left|\int_{a}^{b} f(x) d x-\frac{1}{2}(b-a)(f(a)+f(b))+\frac{5}{4}(b-a)^{2}\left(f^{\prime}(b)-f^{\prime}(a)\right)\right| \\
\leq & \frac{(b-a)^{3}}{4}\left(\frac{7}{3}\right)^{\frac{p-1}{p}}\left\{\left(\frac{4 s^{2}+16 s+14}{s^{3}+6 s^{2}+11 s+6}\left|f^{\prime \prime}(a)\right|^{p}+\frac{s^{2}+7 s+14}{s^{3}+6 s^{2}+11 s+6}\left|f^{\prime \prime}(b)\right|^{p}\right)^{\frac{1}{p}}\right. \\
& \left.+\left(\frac{4 s^{2}+16 s+14}{s^{3}+6 s^{2}+11 s+6}\left|f^{\prime \prime}(b)\right|^{p}+\frac{s^{2}+7 s+14}{s^{3}+6 s^{2}+11 s+6}\left|f^{\prime \prime}(a)\right|^{p}\right)^{\frac{1}{p}}\right\} .
\end{aligned}
$$

Proof. Using Lemma ??, Definition ?? and power mean integral inequality, we obtain

$$
\begin{aligned}
& \left|\int_{a}^{b} f(x) d x-\frac{1}{2}(b-a)(f(a)+f(b))+\frac{5}{4}(b-a)^{2}\left(f^{\prime}(b)-f^{\prime}(a)\right)\right| \\
& \leq \frac{(b-a)^{3}}{4} \int_{0}^{1}|t+1|^{2}\left|f^{\prime \prime}(t a+(1-t) b)+f^{\prime \prime}(t b+(1-t) a)\right| d t \\
& \leq \frac{(b-a)^{3}}{4}\left(\int_{0}^{1}|t+1|^{2} d t\right)^{1-\frac{1}{p}} \\
& \left\{\left(\int_{0}^{1}(t+1)^{2}\left(t^{s}\left|f^{\prime \prime}(a)\right|^{p}+(1-t)^{s}\left|f^{\prime \prime}(b)\right|^{p}\right) d t\right)^{\frac{1}{p}}\right. \\
& \left.+\left(\int_{0}^{1}(t+1)^{2}\left(t^{s}\left|f^{\prime \prime}(b)\right|^{p}+(1-t)^{s}\left|f^{\prime \prime}(a)\right|^{p}\right) d t\right)^{\frac{1}{p}}\right\} \\
& \leq \frac{(b-a)^{3}}{4}\left(\frac{7}{3}\right)^{1-\frac{1}{p}}\left\{\left(\left|f^{\prime \prime}(a)\right|^{p} \int_{0}^{1}(t+1)^{2} t^{s} d t+\left|f^{\prime \prime}(b)\right|^{p} \int_{0}^{1}(t+1)^{2}(1-t)^{s} d t\right)^{\frac{1}{p}}\right. \\
& \left.+\left(\left|f^{\prime \prime}(b)\right|^{p} \int_{0}^{1}(t+1)^{2} t^{s} d t+\left|f^{\prime \prime}(a)\right|^{p} \int_{0}^{1}(t+1)^{2}(1-t)^{s} d t\right)^{\frac{1}{p}}\right\} \\
& \leq \frac{(b-a)^{3}}{4}\left(\frac{7}{3}\right)^{1-\frac{1}{p}} \\
& \times\left\{\left(\frac{4 s^{2}+16 s+14}{s^{3}+6 s^{2}+11 s+6}\left|f^{\prime \prime}(a)\right|^{p}+\frac{s^{2}+7 s+14}{s^{3}+6 s^{2}+11 s+6}\left|f^{\prime \prime}(b)\right|^{p}\right)^{\frac{1}{p}}\right. \\
& +\left(\frac{4 s^{2}+16 s+14}{s^{3}+6 s^{2}+11 s+6}\left|f^{\prime \prime}(b)\right|^{p}+\frac{s^{2}+7 s+14}{s^{3}+6 s^{2}+11 s+6}\left|f^{\prime \prime}(a)\right|^{p}\right)^{\frac{1}{p}}
\end{aligned}
$$

The proof is done.

Theorem 11. Let $f: I^{\circ} \subseteq R \rightarrow R$ be a differentiable mapping on $I^{\circ}, a, b \in I^{\circ}$ with $a<b$, and let $p>1$ with $1 / p+1 / q=1$. If the mapping $\left|f^{\prime \prime}\right|^{p}$ is $\operatorname{tgs}$-convex on $[a, b]$, then the following inequality 
holds:

$$
\begin{aligned}
& \quad\left|\int_{a}^{b} f(x) d x-\frac{1}{2}(b-a)(f(a)+f(b))+\frac{5}{4}(b-a)^{2}\left(f^{\prime}(b)-f^{\prime}(a)\right)\right| \\
& \leq \frac{7(b-a)^{3}}{6}\left(\frac{23}{140}\right)^{\frac{1}{p}}\left(\left|f^{\prime \prime}(a)\right|^{p}+\left|f^{\prime \prime}(b)\right|^{p}\right)^{\frac{1}{p}} .
\end{aligned}
$$

Proof. Using Lemma ??, Definition ?? and power mean integral inequality, we obtain

$$
\begin{aligned}
& \left|\int_{a}^{b} f(x) d x-\frac{1}{2}(b-a)(f(a)+f(b))+\frac{5}{4}(b-a)^{2}\left(f^{\prime}(b)-f^{\prime}(a)\right)\right| \\
\leq & \frac{(b-a)^{3}}{4} \int_{0}^{1}|t+1|^{2}\left|f^{\prime \prime}(t a+(1-t) b)+f^{\prime \prime}(t b+(1-t) a)\right| d t \\
\leq & \frac{(b-a)^{3}}{4}\left(\int_{0}^{1}|t+1|^{2} d t\right) \\
& \left\{\left(\int_{0}^{1}(t+1)^{2}\left(t(1-t)\left|f^{\prime \prime}(a)\right|^{p}+t(1-t)\left|f^{\prime \prime}(b)\right|^{p}\right) d t\right)^{\frac{1}{p}}\right. \\
& \left.+\left(\int_{0}^{1}(t+1)^{2}\left(t(1-t)\left|f^{\prime \prime}(b)\right|^{p}+t(1-t)\left|f^{\prime \prime}(a)\right|^{p}\right) d t\right)^{\frac{1}{p}}\right\} \\
\leq & \frac{(b-a)^{3}}{2}\left(\frac{7}{3}\right)^{1-\frac{1}{p}}\left(\left|f^{\prime \prime}(a)\right|^{p}+\left|f^{\prime \prime}(b)\right|^{p}\right)^{\frac{1}{p}}\left(\int_{0}^{1}(t+1)^{2} t(1-t) d t\right)^{\frac{1}{p}} \\
\leq & \frac{7(b-a)^{3}}{6}\left(\frac{23}{140}\right)^{\frac{1}{p}}\left(\left|f^{\prime \prime}(a)\right|^{p}+\left|f^{\prime \prime}(b)\right|^{p}\right)^{\frac{1}{p}} .
\end{aligned}
$$

The proof is completed.

\section{Applications to Secial Means}

Now we shall use the results of Section 2 to prove the following new inequalities connecting the above means for arbitrary real numbers.

Proposition 1. Let $a, b \in R, 0<a<b$ and $n \geq 2$. Then, the following inequality holds:

$$
\begin{aligned}
& \left|(b-a) L_{n}(a, b)-\frac{b-a}{2} A\left(a^{n}, b^{n}\right)-\frac{5 n}{4}(b-a)^{2}\left(b^{n-1}-a^{n-1}\right)\right| \\
\leq & \frac{7}{6}(b-a)^{3} n(n-1) A\left(a^{n-2}, b^{n-2}\right) .
\end{aligned}
$$

Proof. The proof is immediate from Theorem ?? applied for $f(x)=x^{n}, x \in R$. 
Proposition 2. Let $a, b \in R, s \in(0,1], 0<a<b$. Then, the following inequality holds:

$$
\begin{aligned}
& \left|(b-a) L_{s}(a, b)-\frac{b-a}{2} A\left(a^{s}, b^{s}\right)-\frac{5 s}{4}(b-a)^{2}\left(b^{s-1}-a^{s-1}\right)\right| \\
\leq & s(1-s) \frac{(b-a)^{3}}{2} \frac{\left(5 s^{2}+23 s+28\right)}{s^{3}+6 s^{2}+11 s+6} A\left(a^{s-2}, b^{s-2}\right) .
\end{aligned}
$$

Proof. The proof is immediate from Theorem ?? applied for $f(x)=x^{s}, x \in R$ and $s \in(0,1)$.

Proposition 3. Let $a, b \in R, 0<a<b$, and $n \in N, n>2$. Then, for all $p>1$, the following inequality holds:

$$
\begin{aligned}
& \left|(b-a) L_{n}(a, b)-\frac{b-a}{2} A\left(a^{n}, b^{n}\right)-\frac{5 n}{4}(b-a)^{2}\left(b^{n-1}-a^{n-1}\right)\right| \\
\leq & n(n-1) \frac{(b-a)^{3}}{2}\left(\frac{2^{2 p+1}-1}{2 p+1}\right)^{1 / p} A^{(p-1) / p}\left(a^{\frac{(n-2) p}{p-1}}, b^{\frac{(n-2) p}{p-1}}\right) .
\end{aligned}
$$

Proof. The proof is immediate from Theorem ?? applied for $f(x)=x^{n}, x \in R$.

Proposition 4. Let $a, b \in R, s \in(0,1), 0<a<b$. Then, for all $p, q>1$, the following inequality holds:

$$
\begin{aligned}
& \left|(b-a) L_{s}(a, b)-\frac{b-a}{2} A\left(a^{s}, b^{s}\right)-\frac{5 s}{4}(b-a)^{2}\left(b^{s-1}-a^{s-1}\right)\right| \\
\leq & s(1-s) \frac{(b-a)^{2}}{4}\left(\frac{2^{2 p+1}-1}{2 p+1}\right)^{1 / p}\left\{\left(\frac{a^{q(s-2)}}{s+1}+b^{q(s-2)} \frac{\Gamma(s+1)}{\Gamma(s+2)}\right)^{\frac{1}{q}}\right. \\
+ & \left.\left(\frac{b^{q(s-2)}}{s+1}+a^{q(s-2)} \frac{\Gamma(s+1)}{\Gamma(s+2)}\right)^{\frac{1}{q}}\right\}
\end{aligned}
$$

Proof. The proof is immediate from Theorem ?? applied for $f(x)=x^{s}, x \in R$ and $s \in(0,1)$.

Proposition 5. Let $a, b \in R, 0<a<b$, and $0 \neq[a, b]$. Then, for all $p>1$, the following inequality holds:

$$
\begin{aligned}
& \left|\frac{5}{2}(b-a)^{2} \frac{A(a, b)}{G^{4}(a, b)}-H^{-1}(a, b)+L^{-1}(a, b)\right| \\
\leq & \frac{(b-a)^{2} \cdot 7^{\frac{p-1}{p}}}{4^{\frac{p+1}{p}} .3}\left\{\left(17\left(\frac{2}{a^{3}}\right)^{p}+11\left(\frac{2}{b^{3}}\right)^{p}\right)^{1 / p}+\left(17\left(\frac{2}{b^{3}}\right)^{p}+11\left(\frac{2}{a^{3}}\right)^{p}\right)^{1 / p}\right\}
\end{aligned}
$$

Proof. The proof is immediate from Theorem ?? applied for $f(x)=\frac{1}{x}, x \in[a, b]$. 
Proposition 6. Let $a, b \in R, s \in(0,1), 0<a<b$, and $[a, b] \neq 0$. Then, for all $p>1$, the following inequality holds:

$$
\begin{aligned}
& \left|L^{-1}(a, b)-H^{-1}\left(a^{s}, b^{s}\right)+\frac{5 s(b-a)\left(b^{s+1}-a^{s+1}\right)}{4 G^{2(s+1)}(a, b)}\right| \\
\leq & \frac{(b-a)^{2} s(s+1)}{4}\left(\frac{7}{3}\right)^{1-\frac{1}{p}}\left\{\left(\frac{4 s^{2}+16 s+14}{s^{3}+6 s^{2}+11 s+6}\left(\frac{1}{a^{s+2}}\right)^{p}+\frac{s^{2}+7 s+14}{s^{3}+6 s^{2}+11 s+6}\left(\frac{1}{b^{s+2}}\right)^{p}\right)^{\frac{1}{p}}\right. \\
& \left.+\left(\frac{4 s^{2}+16 s+14}{s^{3}+6 s^{2}+11 s+6}\left(\frac{1}{b^{s+2}}\right)^{p}+\frac{s^{2}+7 s+14}{s^{3}+6 s^{2}+11 s+6}\left(\frac{1}{a^{s+2}}\right)^{p}\right)^{\frac{1}{p}}\right\}
\end{aligned}
$$

Proof. The proof is immediate from Theorem ?? applied for $f(x)=\frac{1}{x^{s}}, x \in R$ and $s \in(0,1)$.

\section{References}

[1] M. Alomari, M. Darus, U.S. Kırmac1, Some Inequalities of Hermite-Hadamard type for $s$ convex Functions, Acta Math. Sci. 31B(4), (2011)1643-1652.

[2] P. Burai, A. Házy, and T. Juhász, Bernstein-Doetsch type results for s-convex functions, Publ. Math. Debrecen 75 (2009), no. 1-2, 23-31.

[3] P. Burai, A. Házy, and T. Juhász, On approximately Breckner s-convex functions, Control Cybernet. 40 (2011), no. 1, 91-99.

[4] P. Cerone, On perturbed trapezoidal and midpoint rules, Korean J. Comput. Appl. Math., 2 (2002), 423-435

[5] S.S. Dragomir and R.P. Agarwal, Two inequalities for differentiable mappings and applications to special means of real numbers and to trapezoidal formula, Appl. Math. Lett., 11(5) (1998), 91-95.

[6] S.S. Dragomir, P. Cerone and A. Sofo, Some remarks on the trapezoid rule in numerical integration, Indian J. Pure Appl. Math., 31(5) (2000), 475-494.

[7] S.S. Dragomir, Y.J. Cho and S.S. Kim, Inequalities of Hadamard's type for Lipschitizian mappings and their applications, J. Math. Anal. Appl., 245 (2000), 489-501.

[8] S.S. Dragomir and S. Wang, An inequality of Ostrowski-Grüss' type and its applications to the estimation of error bounds for some special means and for some numerical quadrature rules, Computers Math. Applic., 33(11) (1997), 15-20.

[9] S.S. Dragomir and S. Wang, Applications of Ostrowski' inequality to the estimation of error bounds for some special means and for some numerical quadrature rules, Appl. Math. Lett., 11(1) (1998), 105-109. 
[10] S.S. Dragomir, J.E. Pečarić and J. Săndor, A note on the Jensen-Hadamard's inequality, Anal. Num. Ther. Approx. 19, 29-34 (1990).

[11] S.S. Dragomir, Two mappings in connection to Hadamard's inequality, J. Math. Anal. Appl. 167, 49-56 (1992).

[12] S.S. Dragomir, S. Fitzpatrick, The Hadamard's inequality for s-convex functions in the second sense, Demonstration Math., 32 (4) (1999), 687-696.

[13] J. Hadamard, Étude sur les propriétés des fonctions entières en particulier d'une fonction considérée par Riemann, J. Math. Pures Appl. 58 (1893) 171-215.

[14] H. Hudzik and L. Maligranda, Some remarks on s-convex functions, Aequationes Math., 48, 100-111, (1994).

[15] D. S. Mitrinović, J. Pečarić, and A.M. Fink, Classical and new inequalities in analysis, KluwerAcademic, Dordrecht, 1993.

[16] J.E. Pečarić , F. Proschan and Y.L. Tong, Convex Functions, Partial Ordering and Statistical Applications, Academic Press, New York, (1991).

[17] M. Tunç, E. Göv, Ü. Şanal, On tgs-convex function and their inequalities, Facta Univ. Ser. Math. Inform. Accepted

[18] B.-Y. Xi and F. Qi, Some Integral Inequalities of Hermite-Hadamard Type for Convex Functions with Applications to Means, Journal of Function Spaces and Appl., Volume 2012, Article ID 980438, 14 p., doi:10.1155/2012/980438.

[19] G.S. Yang, D.Y. Hwang, K.L. Tseng, Some inequalities for differentiable convex and concave mappings, Comput. Math. Appl. 47 (2004) 207-216. 